\title{
Diabetes, Intracranial Stenosis and Microemboli in Asymptomatic Carotid Stenosis
}

\author{
Thach D. Lam, Stephanie Lammers, Claudio Munoz, Arturo Tamayo, \\ J. David Spence
}

\begin{abstract}
Background: The risk of stroke in patients with asymptomatic carotid stenosis (ACS) is now so low that it is important to have methods to identify those patients most likely to benefit from intervention, or who may require special consideration in choice of medical therapy. We studied the prediction of stroke, death or transient ischemic attacks (stroke/death/TIA) in patients with ACS by intracranial arterial stenosis, and microemboli on transcranial Doppler (TCD), and the effect of diabetes mellitus on microemboli, intracranial stenosis and risk of events. Methods: Patients with ACS $>60 \%$ by Doppler ultrasound were recruited from the Stroke Prevention Clinic of University Hospital, London, Canada. All 339 participants underwent TCD for detection of intracranial stenosis and detection of microemboli, and carotid ultrasound to measure extracranial stenosis and total carotid plaque area. Participants were followed for three years, to determine the risk of stroke/death/TIA. Results: Stroke/death/TIA occurred in $38 \%$ of patients with microemboli versus $10 \%$ without $(\mathrm{p}=0.0001$ ), and in $18 \%$ of patients with intracranial stenosis, versus $10 \%$ without ( $\mathrm{p}=0.042$ ). Diabetics were significantly more likely to have intracranial stenosis (45\% vs. 29\%, p =0.014), microemboli $(38 \%$ vs. 10\%, p <0.0001), and had significantly higher risk of stroke/death/TIA over three years $(21 \%$ vs. $11 \%$ without; $\mathrm{p}=0.024)$. Survival free of stroke, TIA or death was significantly better without microemboli or intracranial stenosis $(\mathrm{p}<0.0001)$. Conclusions: Diabetes, microemboli and intracranial stenosis predicted higher risk of stroke, death or TIA than did extracranial stenosis or total plaque area; diabetics may need more intensive therapy.
\end{abstract}

RÉSUMÉ: Diabète, sténose intracrânienne et microembolies dans la sténose carotidienne asymptomatique. Contexte : Le risque d'accident vasculaire cérébral (AVC) chez les patients porteurs d'une sténose carotidienne asymptomatique (SCA) est maintenant si faible qu'il est important d'établir des critères pour identifier les patients qui sont les plus susceptibles de bénéficier d'une intervention ou chez qui on doit porter une attention particulière au choix du traitement médical. Nous avons examiné si la présence d'une sténose artérielle intracrânienne et de microembolies au Doppler transcrânien (DTC) étaient des facteurs de prédiction de l'AVC, du décès ou d'accès ischémiques transitoires (AIT) (AVC/décès/AIT) chez des patients porteurs d'une SCA ainsi que l'effet du diabète sur les microembolies, la sténose intracrânienne et le risque d'événements. Méthode : Nous avons recruté des patients présentant une SCA > 60\% à l'échographie Doppler à la clinique de prévention de l'AVC à l'hôpital universitaire de London, en Ontario, au Canada. Les 339 participants ont subi un DTC pour la détection de la sténose intracrânienne et de microembolies et une échographie carotidienne pour mesurer la sténose extracrânienne et la surface totale de plaque. Les participants ont été suivis pendant 3 ans afin de déterminer le risque d'AVC/décès/AIT. Résultats : Un AVC/décès/AIT est survenu chez 38\% des patients avec microembolies et chez 10\% de ceux qui n'en avaient pas (p $=0,0001)$ ainsi que chez $18 \%$ des patients porteurs d'une sténose intracrânienne et chez $10 \%$ de ceux qui n'en avaient pas $(p=0,042)$. Les diabétiques étaient significativement plus susceptibles de présenter une sténose intracrânienne que ceux qui n'étaient pas diabétiques (45\% par opposition à 29\%; $\mathrm{p}$ $=0,014)$, des microembolies $(38 \%$ par opposition à $10 \% ; \mathrm{p}<0,0001)$ et ils avaient un risque significativement plus élevé d'AVC/décès/AIT au cours des trois années d'observation (21\% par opposition à $11 \% ; \mathrm{p}=0,024)$. La survie sans AVC, AIT ou décès était significativement meilleure sans microembolies ou sténose intracrânienne ( $<$ 0,0001). Conclusions : Le diabète, les microembolies et la sténose intracrânienne prédisaient un risque plus élevé d'AVC, de décès ou d'AIT que la sténose extracrânienne ou la surface totale de plaque. Il se peut que les diabétiques aient besoin d'un traitement plus énergique.

Can J Neurol Sci. 2013; 40: 177-181

With more intensive medical therapy, the risk of stroke or death in patients with asymptomatic carotid stenosis is now so low that most patients will not benefit from endarterectomy or stenting ${ }^{1}$. It is therefore important to have methods to identify the small proportion who could benefit from intervention ${ }^{1,2}$, or who may require special consideration in choice of medical therapy. We have previously reported that carotid plaque area ${ }^{3}$, microemboli on transcranial Doppler ${ }^{4,5}$ (TCD), and carotid plaque ulceration on 3-dimensional ultrasound ${ }^{5}$ predict the risk of cardiovascular events, particularly of stroke. The predictive value of TCD microembolus detection in patients with asymptomatic carotid stenosis was confirmed in 2010 by Markus et $\mathrm{al}^{6}$.

From the Schulich School of Medicine and Dentistry (TDL, SL, JDS), Stroke Prevention and Atherosclerosis Research Centre, Robarts Research Institute (CM, AT, JDS), University of Western Ontario, London; Queen's University School of Medicine (SL), Kingston, Ontario; 4. Brandon Health Sciences Centre (AT), Brandon, Manitoba, Canada.

Received September 13, 2012. Final Revisions Submitted October 30, 2012 Correspondence to: David Spence, Stroke Prevention and Atherosclerosis Research Centre, Siebens-Drake Building, Robarts Research Institute, 1400 Western Road, London, Ontario, N6G 2V2, Canada. Email: dspence@ robarts.ca. 
It is widely believed that intracranial arterial stenosis is biologically and genetically different from extracranial carotid stenosis. For example, the burden of intracranial stenosis is especially high among Asians, Africans and Hispanics ${ }^{7}$, whereas extracranial stenosis is a more common cause of stroke in Caucasian populations in Europe and America ${ }^{8}$. Pathophysiologically, the occurrence of intracranial stenosis has been primarily attributed to age, diabetes mellitus and hypertension ${ }^{9}$, while hyperlipidemia has been identified as an independent risk factor for extracranial stenosis ${ }^{10}$.

In this study, we examined the prediction of stroke, death, transient ischemic attack (TIA) and myocardial infarction by diabetes, intracranial arterial stenosis, microemboli, extracranial stenosis of the internal carotid, and total plaque area. We also studied the effect of diabetes mellitus on microemboli, intracranial stenosis and risk of events.

\section{Methods}

\section{Study population}

All patients were recruited from the Stroke Prevention Clinic or the Premature Atherosclerosis Clinic of University Hospital, London, Canada, beginning in 2000 for a prospective peerreviewed study to investigate whether the frequency of microemboli in the intracerebral arteries, as evaluated by Transcranial Doppler (TCD) testing, predicted stroke risk ${ }^{11}$. Patients with atrial fibrillation, prosthetic valves or other cardiac sources of emboli were excluded. All participants consented to a protocol approved by the University of Western Ontario Human Subjects Research Ethics Board. Data were analyzed for all patients who had a TCD study performed at our Stroke Prevention and Atherosclerosis Research Centre (SPARC) in London, Ontario, and for whom data were available on plaque area, intracranial stenosis, microemboli and vascular risk factors.

\section{Assessment of baseline characteristics}

Baseline data were obtained at the patient's initial visit at the SPARC. Risk factors included age, sex, diabetes, systolic blood pressure, diastolic blood pressure, total cholesterol, triglycerides, HDL cholesterol, LDL cholesterol, total homocysteine (tHcy) and smoking history (pack-years). We also recorded use of antihypertensive therapy, use of lipid-lowering medication, and medical history of TIA, ischemic stroke and myocardial infarction (MI). Based on the rate of decline of events among patients in the North American Symptomatic Carotid Endarterectomy Trial (NASCET) ${ }^{12}$, patients were regarded as asymptomatic if they had experienced a TIA more than 18 months before enrolment in the study. Diabetes mellitus was classified in subjects with fasting glucose $\geq 7.0 \mathrm{mmol} / \mathrm{L}$, random glucose $\geq 11.1 \mathrm{mmol} / \mathrm{L}$, or use of medication for glycemic control. Blood pressures were obtained from each arm while the patient was in a seated position using an automated blood pressure device (DINAMAPP in earlier years, BPTru since 2006). The blood pressure recorded in the database was that in the arm with the higher pressure. Laboratory examinations included total serum cholesterol, triglycerides, HDL cholesterol, LDL cholesterol, and plasma total homocysteine after an overnight fast. These tests were performed using routine methods in the Biochemistry laboratory of University Hospital.

\section{Assessment of stenosis type and total carotid plaque area Duplex Ultrasound}

All patients underwent a baseline measurement of total carotid plaque area and extracranial carotid stenosis, as previously described ${ }^{3}$. Briefly, all plaques seen in the common, internal, and external carotids on both sides of the body were measured by duplex sonography. Plaque was measured with an ATL/Phillips HDI 5000 duplex scanner with compound imaging (SonoCT; Phillips, Andover, Massachusetts). The cross-sectional area of each plaque was measured in a longitudinal view in the plane where the plaque area was greatest; the total of the crosssectional areas of all plaques was carotid total plaque area. The use of carotid total plaque area to assess atherosclerotic burden and predict cardiovascular risk has been previously described ${ }^{13}$ and validated ${ }^{3}$. Percent stenosis in each carotid artery was measured by converting the peak frequency in each internal carotid artery into percentage stenosis. Extracranial stenosis was diagnosed if there was a stenosis of $60 \%$ or greater in the left or right internal carotid artery, based on a peak velocity $>170$ $\mathrm{cm} / \mathrm{sec}$. This cut-off was validated angiographically for our laboratory in the ACAS study ${ }^{14}$. Patients were categorized as having or not having extracranial stenosis.

\section{Transcranial Doppler}

All patients underwent a routine TCD study to identify intracranial stenosis in the middle cerebral artery within two weeks of referral to the Stroke Prevention Clinic, using a $2 \mathrm{MHz}$ probe, in power M-mode with a digital TCD system (Spencer Technologies Inc.). Transcranial Doppler procedures were performed on all patients in the semi-recumbent position with a pillow beneath the head. Intracranial stenosis was diagnosed if there was a stenosis of $50 \%$ or greater of a middle cerebral artery; this was defined by a mean systolic velocity of $100 \mathrm{~cm} / \mathrm{s}$ in the absence of a generalized hyperdynamic state. This criterion was validated by Felberg et al for the detection of middle cerebral artery stenosis with $\mathrm{TCD}^{15}$, and confirmed recently by Zhao et $\mathrm{al}^{16}$. Patients were categorized as having or not having intracranial stenosis.

This was followed by monitoring of both middle cerebral arteries, preferably in the M1 segment, through a posterior or middle temporal window. Middle cerebral arteries were identified bilaterally within depths of insonation between 35 to $56 \mathrm{~mm}$ from the temporal window and monitored for up to one hour on two occasions a week apart using a Spencer Mark 500 head-fixation device. Because the headgear is somewhat uncomfortable, and because some patients had difficulty lying still for long periods, monitoring was stopped in some cases after at least 40 minutes, or if the test was positive, exhibiting more than 2 microemboli ipsilateral to the stenosed carotid artery.

Microembolic signals were defined by unidirectionality, duration of $<300 \mathrm{~ms}$, and intensity of $>8 \mathrm{~dB}$ above the Doppler background, with adjustment of gain to enhance detection; settings for microemboli detection were: leading cols $255 \mathrm{~mm}$, trailing cols $255 \mathrm{~mm}$, microemboli-threshold $9 \mathrm{~mm}$, and rejection $55 \mathrm{~mm}$, corresponding to international consensus recommendations ${ }^{17}$. Monitoring was performed and analyzed by one of two observers (AT or CM). All sessions were recorded on the hard drive for review and confirmation of microembolic signals noted during monitoring. 


\section{Statistical analyses}

Clinical and demographic data were compared among patients with different stenosis types and microemboli. Statistical analyses were performed with IBM SPSS Statistics version 20 (SPSS Inc., Chicago, Illinois). Analysis of variance was used to compare continuous variables across the stenosis groups. Pearson's $\chi^{2}$ test was used to compare categorical variables across the stenosis groups; Exact Chi-Square values were computed asymptotically. Cox proportional hazards regression analysis of factors predicting stroke, death or transient ischemic attack (stroke/death/TIA) was performed in a backward Wald analysis. The primary outcome was time to stroke/death/ TIA in a Kaplan-Meier analysis.

\section{Results}

There were 339 patients with complete data. Of the total, mean age \pm standard deviation was $70 \pm 9$ years, mean smoking history was $25 \pm 25$ pack-years, $35 \%$ were female, $17 \%$ had diabetes mellitus, $82 \%$ were on hypertension therapy, $82 \%$ were on lipid therapy. A history of TIA more than 18 months before enrolment in the study was present in $22 \%$ and $22 \%$ had experienced a myocardial infarction. (Baseline characteristics are presented in the Table, divided into groups by stenosis type.) Intracranial stenosis was present in 112 patients (33\%); of these, the intracranial stenosis was bilateral in $28(25 \%)$. Occlusion of one internal carotid was present in 55 (16\%) cases.

Patients with intracranial stenosis were more likely to be diabetic $(25 \%$ vs. $15 \%, \mathrm{p}=0.014)$ to have microemboli $(16 \%$ vs. $6 \%, \mathrm{p}=0.011)$ and to have a higher level of tHcy $(12 \pm 6$ vs. 10 $\pm 5 \mu \mathrm{mol} / \mathrm{L}, \mathrm{p}=0.002)$. Patients with diabetes were significantly more likely to have intracranial stenosis $(45 \%$ vs. $29 \%, \mathrm{p}$ $=0.014)$, microemboli ( $38 \%$ vs. $10 \%, \mathrm{p}<0.0001$ ), and had significantly higher risk of stroke/death/TIA over three years
( $21 \%$ vs. $11 \%$ without; $\mathrm{p}=0.024)$. Time to events was also significantly less for patients with diabetes in a Kaplan-Meier survival analysis $(\log$ rank $\mathrm{p}=0.013)$. Patients with microemboli had significantly higher levels of total homocysteine (16 $\pm 10 \mathrm{vs.}$ $10 \pm 5 \mu \mathrm{mol} / \mathrm{L}, \mathrm{p}=0.0001)$. Survival free of stroke, TIA or death was significantly better without microemboli or intracranial stenosis $(\mathrm{p}<0.0001)$.

Carotid plaque area was also significantly different among the different types of stenosis groups (ANOVA; $p=0.046$; Table 1). Patients with intracranial stenosis were significantly more likely to have higher carotid total plaque area (TPA) compared to patients with no intracranial stenosis $(333 \pm 172$ vs. $291 \pm 140$ $\mathrm{mm}^{2}, \mathrm{p}=0.017$ ), and patients with microemboli had significantly higher TPA compared to patients without microemboli (357 \pm 154 vs. $299 \pm 152 \mathrm{~mm}^{2}, \mathrm{p}=0.037$ ).

The Figure shows the Kaplan-Meier survival plot for survival free of stroke, death or TIA. Time to stroke/death/TIA over three years was significantly less among patients with intracranial stenosis (logrank $\mathrm{p}=0.04$ ) and with microemboli (logrank $\mathrm{p}=0.0001$ ). The survival analysis for stroke or death over three years gave the same result (online supplemental Figure 1). Remote history of TIA did not predict events: stroke/death/TIA occurred among $13 \%$ of patients without a prior history of TIA versus $12 \%$ of those with a remote history of TIA $(\mathrm{p}=0.51)$.

Cox regression analysis of the three-year risk of stroke/death/ TIA was carried out with age, sex, systolic blood pressure, total cholesterol, triglycerides, HDL cholesterol, LDL cholesterol, total homocysteine, total plaque area, smoking status, diabetes, intracranial stenosis and microemboli as variables in the model. In the final step (step 9) of a backward Wald analysis, the variables that entered were age $(\mathrm{p}=0.0433)$, triglycerides $(\mathrm{p}=0.001)$, microemboli $(\mathrm{p}=0.0001)$ and diabetes $(\mathrm{p}=0.054)$. When total homocysteine level was added to the model, only age $(\mathrm{p}=0.03)$ and diabetes $(\mathrm{p}=0.03)$ remained significant in step 11.

Table: Baseline characteristics of study patients by stenosis type

\begin{tabular}{|c|c|c|c|c|}
\hline Characteristics & $\begin{array}{c}\text { Extracranial } \\
\text { only } \\
\mathbf{n}=\mathbf{2 0 6} \\
\end{array}$ & $\begin{array}{c}+ \text { Intracranial } \\
n=99\end{array}$ & $\begin{array}{c}+ \text { Microemboli } \\
n=34\end{array}$ & $\begin{array}{c}\text { +Microemboli } \\
+ \text { intracranial } \\
n=18\end{array}$ \\
\hline Age, $y$ & $70 \pm 9$ & $69+8$ & $70 \pm 7$ & $69+8$ \\
\hline SBP, mm Hg & $144+22$ & $146+22$ & $142 \pm 18$ & $146 \pm 22$ \\
\hline DBP, mm Hg & $74 \pm 12$ & $73 \pm 11$ & $70 \pm 14$ & $75 \pm 14$ \\
\hline Total plaque area, $\mathbf{m m}^{2}$ & $291 \pm 138$ & $328 \pm 171$ & $354 \pm 159$ & $359 \pm 155$ \\
\hline Total cholesterol, $\mathbf{m m o l} / \mathrm{L}$ & $4.6 \pm \overline{ \pm} 1.0$ & $4.7 \pm 1.1$ & $4.6 \pm 0.9$ & $4.6 \pm 1.0$ \\
\hline Triglycerides, mmol/L & $1.7 \pm 1.5$ & $1.9 \pm 1.4$ & $1.8+1.2$ & $1.8+0.6$ \\
\hline HDL cholesterol, mmol/L & $1.3 \pm 0.4$ & $1.3 \pm 0.5$ & $1.3 \pm 0.4$ & $1.3 \pm 0.4$ \\
\hline LDL cholesterol, mmol/L & $2.5 \pm 0.9$ & $2.6 \pm 1.0$ & $2.6 \pm 0.7$ & $2.5 \pm 0.8$ \\
\hline Total homocysteine, $\mu \mathrm{mol} / \mathrm{L}$ & $9.7 \pm 3.8$ & $11.0 \pm 5.5$ & $16.8+12.5$ & $15.2 \pm 6.5$ \\
\hline Smoking, pack-years & $23.9 \pm 23.9$ & $25.3+25.5$ & $26.9 \pm 26.1$ & $32.7 \pm 29.7$ \\
\hline Male sex & $6 \overline{8 \%} \%$ & $6 \overline{1 \%} \%$ & $5 \overline{9 \%} \%$ & $5 \overline{9 \%} \%$ \\
\hline Diabetes mellitus & $16 \%$ & $25 \%$ & $27 \%$ & $22 \%$ \\
\hline Previous MI & $19 \%$ & $28 \%$ & $29 \%$ & $24 \%$ \\
\hline Remote TIA* & $22 \%$ & $22 \%$ & $29 \%$ & $12 \%$ \\
\hline
\end{tabular}

SBP, systolic blood pressure; DBP, diastolic blood pressure; HDL, high-density lipoprotein; LDL, low-density lipoprotein; MI, Myocardial infarction; TIA, transient ischemic attack; * Remote TIA was more than 18 months before enrolment 


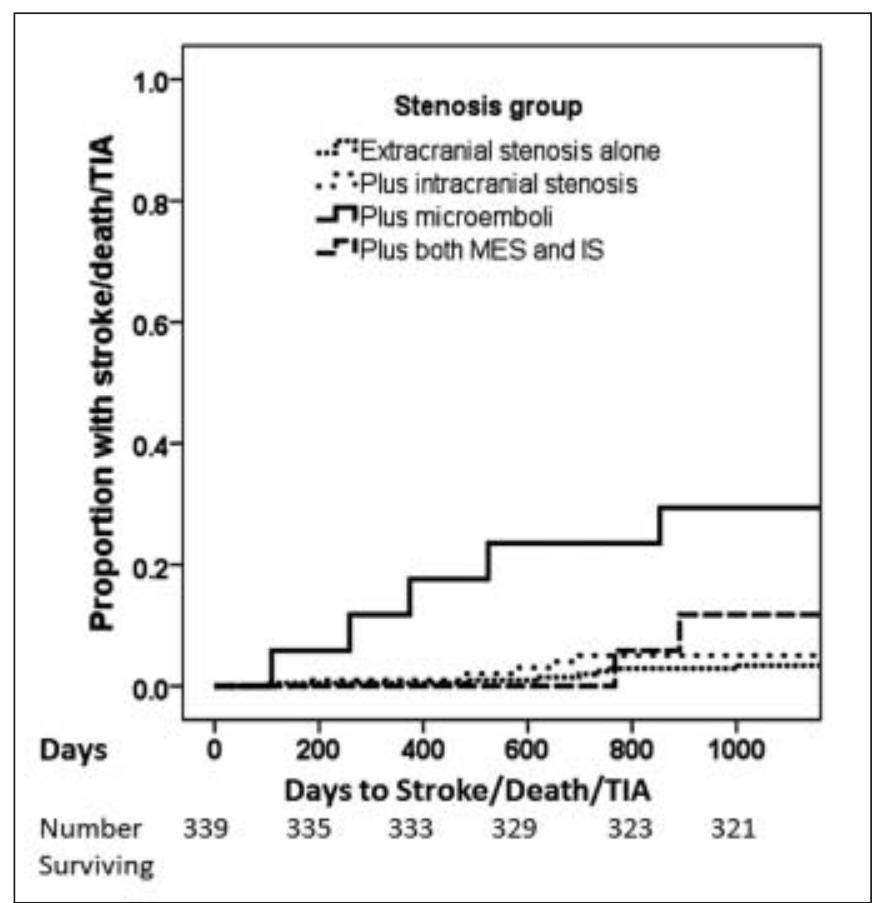

Figure: Kaplan-Meier three-year survival free of stroke, death or TIA by stenosis group. Stroke/death/TIA occurred in 38\% of patients with microemboli versus $10 \%$ without $(p=0.0001)$, and in $18 \%$ of patients with intracranial stenosis, versus $10 \%$ with no stenosis $(p=0.042)$. Event-free survival was significantly worse with intracranial stenosis (logrank $p=0.036$ ) and with two or more microemboli on transcranial Doppler at baseline (logrank $p=0.0001)$. The overall logrank test for the four groups was $p=0.0001$.

\section{Discussion}

We found that in patients with asymptomatic carotid stenosis, microemboli and intracranial stenosis of the middle cerebral artery were stronger predictors of stroke, death or TIA than plaque area or extracranial stenosis of the internal carotid arteries. Survival free of stroke or death gave the same result, but because the occurrence of TIA would usually trigger endarterectomy, we chose to focus on stroke/death/TIA as the primary outcome. Our findings are consistent with a previous study ${ }^{18}$ showing that in patients with asymptomatic intracranial stenosis, the risk of stroke was higher in those with carotid plaque. The three-year risk of stroke, death or TIA was very high with both these phenotypes: $38 \%$ in patients with microemboli and $18 \%$ in patients with intracranial stenosis. The early risk with intracranial stenosis was similar to that with medical therapy in the SAMMPRIS trial ${ }^{19}$, but with longer followup the risk continued to increase. These findings suggest that transcranial Doppler is a powerful tool to identify high-risk patients who warrant more intensive therapy.

We hypothesize that the microemboli detected in the middle cerebral arteries were from the upstream stenosis in the carotid artery. This is supported by the observations of Droste et al, ${ }^{20}$ who reported that microemboli in intracranial stenosis were found only in symptomatic patients with severe stenosis.
Other coronary risk factors (age, sex, blood pressure, cholesterol, smoking) were more strongly related to plaque area than to either intracranial or extracranial stenosis. In this cohort, total plaque area did not predict risk of events, in contrast to our previous report ${ }^{21}$; the reason may be that in this cohort plaque area was much higher on average, and there were few patients with little or no plaque; thus the dynamic range of total plaque area was restricted in this study population (shown in online supplemental Figure 1). This was also true for total extracranial stenosis (online supplemental Figure 2).

Diabetics in our study population had three times the risk of microemboli, and twice the risk of stroke, death or TIA over three years, compared to participants free of diabetes. It is widely accepted that diabetes mellitus increases small-vessel (arteriolar) disease ${ }^{22}$. We found that diabetes mellitus was also associated with increased stenosis in large arteries of the intracranial cerebral vasculature. These findings are consistent with previous reports that showed diabetes mellitus to be preferentially associated with intracranial stenosis. An autopsybased study from Hong Kong reported an increased prevalence of intracranial stenosis in deceased patients with a past medical history of diabetes mellitus ${ }^{23}$. Bae et al showed that in patients with asymptomatic intracranial stenosis, diabetes mellitus was 1.86 times more likely to be present ${ }^{9}$. Kim et al demonstrated that diabetes mellitus was significantly associated with the presence of intracranial stenosis in male subjects (OR 2.09, 95\% CI 1.25-3.51) after adjusting for major confounders ${ }^{24}$. In the Northern Manhattan Stroke Study, a greater occurrence of diabetes mellitus was noted among populations of African or Hispanic descent, which accounted for a major proportion of the increased frequency of intracranial occlusive disease ${ }^{7}$.

There are limitations to our study: the study population consisted of patients referred to a stroke prevention clinic, and with regard to intracranial stenosis, we considered only stenosis in the middle cerebral artery; furthermore, the number of the patients in each subgroup may have limited statistical power. Despite these limitations, we found that intracranial stenosis and microemboli more strongly predicted risk than did total plaque area or extracranial stenosis, and that patients with intracranial stenosis were more likely to be diabetic, and to have microemboli. These findings suggest that patients with intracranial stenosis might warrant more intensive efforts to improve glycemic control, or treatment with drugs that affect insulin resistance, such as pioglitazone, which has been shown to significantly reduce stroke risk in diabetics ${ }^{25}$. Elevated levels of glycosylated hemoglobin strongly predict stroke risk before the onset of diabetes ${ }^{26}$, so in patients with intracranial stenosis and high levels of glycosylated hemoglobin, early treatment with agents such as pioglitazone might be considered. The hypothesis that stroke might be reduced by pioglitazone in patients with stroke and insulin resistance is being tested in the Insulin Resistance Intervention After Stroke (IRIS) study (http://www.iristrial.org/).

\section{ACKNOWLEDGEMENTS}

Carotid duplex scanning was performed by Maria DiCicco RVT and Janine DesRoches RVT. 


\section{SOURCES OF FUNDING}

The study was funded in part by the Heart \& Stroke Foundation of Canada (Ontario), grant numbers NA4990 and T5704. Mr. Lam was supported by the Summer Research Training Program of the Canadian Institutes for Health Research, through the Schulich School of Medicine and Dentistry, Western University.

\section{DISCLOSURES}

Dr. Spence is a member of the Steering Committee, and the country leader for Canada, for the Insulin Resistance Intervention After Stroke (IRIS) study.

\section{REFERENCES}

1. Bogiatzi C, Cocker MS, Beanlands R, Spence JD. Identifying highrisk asymptomatic carotids stenosis. Expert Opin Med Diagnostics. 2012;6:139-51.

2. Cardona P, Rubio F, Martinez-Yelamos S, Krupinski J. Endarterectomy, best medical treatment or both for stroke prevention in patients with asymptomatic carotid artery stenosis. Cerebrovasc Dis. 2007;24 Suppl 1:126-33.

3. Spence JD, Eliasziw M, DiCicco M, Hackam DG, Galil R, Lohmann T. Carotid plaque area: a tool for targeting and evaluating vascular preventive therapy. Stroke. 2002;33: 2916-22.

4. Spence JD, Tamayo A, Lownie SP, Ng WP, Ferguson GG. Absence of microemboli on transcranial Doppler identifies low-risk patients with asymptomatic carotid stenosis. Stroke. 2005;36: 2373-8

5. Madani A, Beletsky V, Tamayo A, Munoz C, Spence JD. High-risk asymptomatic carotid stenosis: ulceration on 3D ultrasound versus TCD microemboli. Neurology. 2011;77:744-50.

6. Markus HS, King A, Shipley M, et al. Asymptomatic embolisation for prediction of stroke in the Asymptomatic Carotid Emboli Study (ACES): a prospective observational study. Lancet Neurol. 2010;9:663-71.

7. Sacco RL, Kargman DE, Gu Q, Zamanillo MC. Race-ethnicity and determinants of intracranial atherosclerotic cerebral infarction. The Northern Manhattan Stroke Study. Stroke. 1995;26:14-20.

8. Wong LK. Global burden of intracranial atherosclerosis. Int $\mathbf{J}$ Stroke. 2006; 1:158-9.

9. Bae HJ, Lee J, Park JM, et al. Risk factors of intracranial cerebral atherosclerosis among asymptomatics. Cerebrovasc Dis. 2007; 24:355-60.

10. Caplan LR, Gorelick PB, Hier DB. Race, sex and occlusive cerebrovascular disease: a review. Stroke. 1986;17:648-55.
11. Spence JD, Coates V, Li H, et al. Effects of intensive medical therapy on microemboli and cardiovascular risk in asymptomatic carotid stenosis. Arch Neurol. 2010;67:180-6.

12. Barnett HJM, Taylor DW, Eliasziw M, et al. Benefit of carotid endarterectomy in patients with symptomatic moderate or severe carotid stenosis. N Engl J Med. 1998;339:1415-25.

13. Spence JD, Hegele RA. Noninvasive phenotypes of atherosclerosis: similar windows but different views. Stroke. 2004;35:649-53.

14. Executive Committee for the Asymptomatic Carotid Atherosclerosis Study. Endarterectomy for asymptomatic carotid artery stenosis. JAMA. 1995;272:1421-8.

15. Felberg RA, Christou I, Demchuk AM, Malkoff M, Alexandrov AV. Screening for intracranial stenosis with transcranial Doppler: the accuracy of mean flow velocity thresholds. J Neuroimaging. 2002;12:9-14.

16. Zhao L, Barlinn K, Sharma VK, et al. Velocity criteria for intracranial stenosis revisited: an international multicenter study of transcranial Doppler and digital subtraction angiography. Stroke. 2011;42:3429-34.

17. Ringelstein EB, Droste DW, Babikian VL, et al. Consensus on microembolus detection by TCD. International Consensus Group on Microembolus Detection. Stroke. 1998;29:725-9.

18. Takahashi W, Ohnuki T, Ide M, Takagi S, Shinohara Y. Stroke risk of asymptomatic intra- and extracranial large-artery disease in apparently healthy adults. Cerebrovasc Dis. 2006;22:263-70.

19. Chimowitz MI, Lynn MJ, Derdeyn CP, et al. Stenting versus aggressive medical therapy for intracranial arterial stenosis. $\mathrm{N}$ Engl J Med. 2011;365:993-1003.

20. Droste DW, Junker K, Hansberg T, Dittrich R, Ritter M, Ringelstein EB. Circulating microemboli in 33 patients with intracranial arterial stenosis. Cerebrovasc Dis. 2002;13:26-30.

21. Spence JD, Eliasziw M, DiCicco M, Hackam DG, Galil R, Lohmann T. Carotid plaque area: a tool for targeting and evaluating vascular preventive therapy. Stroke. 2002;33: 2916-22.

22. Nathan DM. Long-term complications of diabetes mellitus. N Engl J Med. 1993;328:1676-85.

23. Leung SY, Ng TH, Yuen ST, Lauder IJ, Ho FC. Pattern of cerebral atherosclerosis in Hong Kong Chinese. Severity in intracranial and extracranial vessels. Stroke. 1993;24:779-86.

24. Kim BJ, Lee SH, Kang BS, Yoon BW, Roh JK. Diabetes increases large artery diseases, but not small artery diseases in the brain. $\mathrm{J}$ Neurol. 2008;255:1176-81.

25. Wilcox R, Bousser MG, Betteridge DJ, et al. Effects of pioglitazone in patients with type 2 diabetes with or without previous stroke: results from PROactive (PROspective pioglitAzone Clinical Trial In macroVascular Events 04). Stroke. 2007;38:865-73.

26. Selvin E, Steffes MW, Zhu H, et al. Glycated hemoglobin, diabetes, and cardiovascular risk in nondiabetic adults. N Engl J Med. 2010;362:800-11. 